\title{
Régis Dericquebourg (dir.), Le (mal) traitement des nouveaux hérétiques. La France et ses minorités religieuses
}

Paris, L'Harmattan, 2013, 170 p.

Guillaume Roucoux

\section{OpenEdition}

Journals

Édition électronique

URL : http://journals.openedition.org/assr/26364

DOI : $10.4000 /$ assr.26364

ISSN : $1777-5825$

Éditeur

Éditions de l'EHESS

Édition imprimée

Date de publication : 31 décembre 2014

Pagination : 167

ISBN : 978-2-7132-2467-6

ISSN : 0335-5985

\section{Référence électronique}

Guillaume Roucoux, «Régis Dericquebourg (dir.), Le (mal) traitement des nouveaux hérétiques. La France et ses minorités religieuses », Archives de sciences sociales des religions [En ligne], 168 | 2014, mis en ligne le 09 avril 2015, consulté le 22 septembre 2020. URL : http://journals.openedition.org/ assr/26364; DOI : https://doi.org/10.4000/assr.26364 


\section{Régis Dericquebourg (dir.), Le (mal) traitement des nouveaux hérétiques. La France et ses minorités religieuses}

Paris, L'Harmattan, 2013, 170 p.

Guillaume Roucoux

\section{RÉFÉRENCE}

Régis Dericquebourg (dir.), Le (mal) traitement des nouveaux hérétiques. La France et ses minorités religieuses, Paris, L'Harmattan, 2013, 170 p. 
1 Cet ouvrage tient lieu d'actes de la journée d'étude organisée en octobre 2011 par l'Observatoire Européen des Faits Religieux et de la Laïcité, dirigé par le psychosociologue Régis Dericquebourg. Avocats, sociologues et responsables associatifs furent réunis à cette occasion pour montrer chacun à leur manière comment sont «(mal)traités» les membres de «groupes religieux minoritaires» (GRM) dits «sectes» en France, en Belgique et au Japon. Comme le suggère Dericquebourg en introduction, « (mal)traité » pourrait déjà signifier nontraité au regard du faible intérêt que leur accordent les sciences sociales des religions en Europe. Or, ce manque de connaissance actualisée associé au refus d'entendre les universitaires dans le débat public n'est pas sans conséquence sur le (mal)traitement des individus concernés.

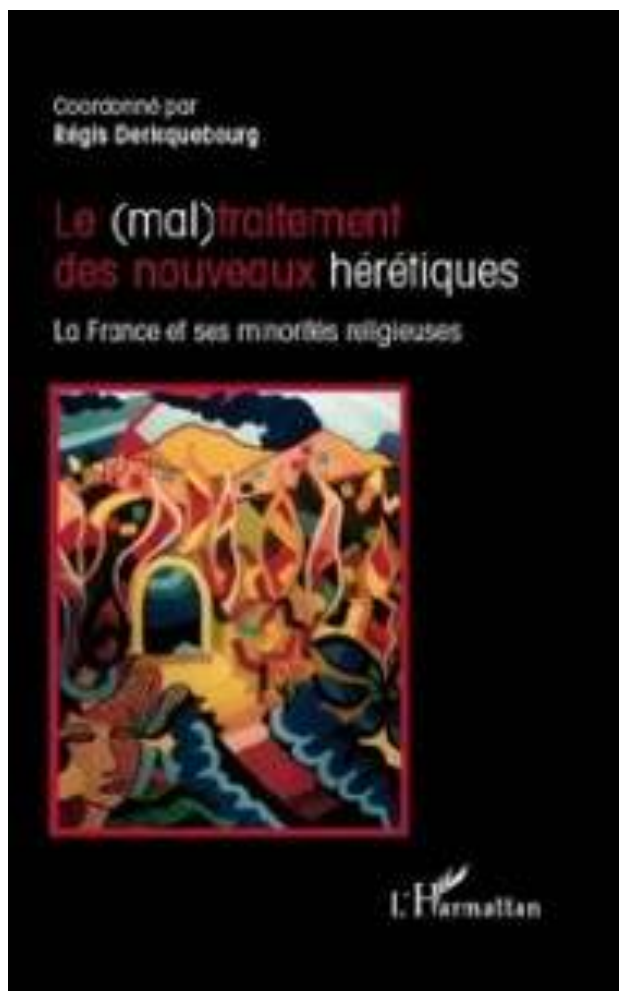
Par exemple en France - qui « a toujours détesté les hérétiques et les marges religieuses » (8) - les associations comme l'Union Nationale des Associations de Défense des Familles et de l'Individu (Unadfi) et la Mission interministérielle de vigilance et de lutte contre les dérives sectaires (Miviludes) ont non seulement réussi à monopoliser la parole d' " expert ", mais elles ont aussi convaincu le gouvernement de subventionner leur lutte, ce que dénoncent plusieurs contributeurs. En ce sens, cet ouvrage se veut résolument critique et engagé contre les entraves faites aux membres de «minorités religieuses » d'exercer leur « liberté de culte ».

2 Sociologue spécialiste des légendes urbaines, Véronique Campion-Vincent analyse le cas hautement médiatisé du village de Bugarach, où se sont rassemblés trente mille individus à l'approche de la «fin du monde " prévue le 21 décembre 2012 (chap. 1). Parmi eux, on comptait aussi la Miviludes accompagnée d'une surveillance renforcée, dont la présence a suscité l'interrogation puisqu'aucun risque n'était identifié. La municipalité ne craignait pas non plus d'atteinte à l'ordre public, seuls les drames passés et la rumeur ont justifié l'inquiétude des agents de la Mission. D'après CampionVincent, la Miviludes existe en raison de trois facteurs spécifiques à la France: le développement d'une culture de la surveillance notamment étatique, des représentations alarmistes ou qui tournent en ridicule lesdites « sectes », et le soutien de la Franc-Maçonnerie. Dans ce contexte « haineusement correct » (Dericquebourg, 2003) généré par la Mission à l'égard des "mouvements religieux hétérodoxes », une approche dépassionnée s'avère d'après l'auteur indispensable pour ne pas succomber à la « paniquesociale».

3 On doit aussi à la Miviludes le concept problématique de «dérive sectaire » qui s'est exporté dans le droit belge où l'avocat Philippe Vanlangendonck l'a étudié (chap.3). Anticonstitutionnel selon lui, il permet à quiconque de «poursuivre 
pénalement n'importe qui pour quasiment n'importe quoi » (p. 60). De fait, sa portée et son " opérationnalisation " furent les premières préoccupations des lecteurs du projet de loi de 2011, qui y voyaient la nécessité de sa "circonscription ». À l'instar du cas français, ce projet fut élaboré comme un dispositif de contrôle social dans le but de sanctionner « l'abus de faiblesse » et de protéger « la personne vulnérable » devenue la cible d'enjeux sociopolitiques, d'un marché lucratif et un prétexte à multiplier les réclamations de justice qui sont autant d'«instrumentalisation[s] pure[s] et simple[s] du législateur» (p. 68). Pour Dericquebourg, la « dérive sectaire » participe d'un système "particratique " et "antidémocratique " qui empêche notamment "l'émergence de nouvelles institutions et associations religieuses et/ou philosophiques pour leur barrer l'accès à la manne des subsides publics qu'il prétend continuer à s'arroger de manière éternelle» (p. 71). La "faiblesse » comme la "sécurité » des "victimes» apparaissent telles des prétextes à la protection de privilèges et à la perpétuation de discriminations, qui menaceraient in fine la démocratie entre autres acquis sociaux. Vanlangendonck reste attentif à la tournure définitive de la loi et des possibles recours au niveau européen, tout en privilégiant à la « dérive » la notion de « risque ».

4 S'agissant encore de la surveillance et l'intervention étatiques, la situation du Japon apparaît inverse à celle de la France et de la Belgique. Directeur de Human Rights Without Frontiers Willy Fautré présente quelques cas de "dé-conversion religieuse " forcée, ignorés des autorités nippones comme des instances internationales en charge du respect des Droits de l'Homme (chap. 2). À partir d'exemples moonistes et jéhovistes, et à l'appui d'une enquête menée par le journaliste Kazuhiro Yonemoto, l'auteur présente comment parents, acteurs antisectes et pasteurs protestants travaillent de concert, dans l'illégalité et le silence général, à cette "privation de liberté " qui implique «enlèvement », « séquestration» et "programme» de «reconversion» (p. 47-48), sans oublier ses coûts élevés et ses conséquences psychologiques importantes. En tant qu'acteur international, Fautré appelle de ses vœux à condamner ces pratiques incompatibles avec les Droits de l'Homme. Il enjoint le Japon à intégrer le droit international en plus d'un volet « religion » dans son système juridique, et recommande enfin d'informer le public, d'engager des enquêtes policières et d'administrer des sanctions.

5 En léger décalage avec les autres chapitres, car il ne traite pas explicitement d' " hérésie ", le sociologue et anthropologue Fabrice Desplan continue sur le thème des droits, en abordant la problématique de leur universalité au regard du recours au religieux - au sens étymologique du terme, qui relie ou crée du lien - dans les projets de reconstruction nationale (chap. 4). Au cœur de son argumentation, Dericquebourg place le concept de «justice transitionnelle» qui donne une "place centrale [à] la victime", souligne le "besoin de paix» et de «justice» en vue de rétablir la démocratie (p. 82). L'auteur observe dans ce projet que "[1]a religion demeure très fonctionnelle» (p. 86) et que «le religieux ne peut pas garantir l'universalité » (p. 88), qui relève, elle, uniquement de la fonction des politiciens.

6 Dans une perspective tout aussi pragmatiste en lien avec le droit, l'avocat Alain Garay, dont le travail porte sur les "procès intentés aux groupes religieux minoritaires", rappelle combien la parole des experts, psychiatres le plus souvent, mais aussi politiques et/ou militants, exceptionnellement chercheurs en sciences sociales, y est déterminante (chap. 5). Même si l'auteur se montre réfractaire à l'idée d'appliquer les 
définitions et typologies sociologiques stricto sensu en droit, il invite "juristes et autres » à se les « réapproprier ». D'après lui, le rôle des experts doit se restreindre à "résoudre une question de fait» le plus objectivement possible, sans répondre aux questions sociologiques, ni juger et encore moins punir. Il souligne enfin la nécessité de pouvoir recourir à une " contre-expertise », afin de mener un " procès équitable ».

Poursuivant sur le thème de l'expertise, Dericquebourg montre en s'appuyant sur l'analyse d'ouvrages antisectes comment le traitement psychologique des hérétiques a rejoint leur traitement politique (chap. 6). Le corpus étudié a pour particularité de se fonder sur les discours des membres d'associations de "victimes" que les interprétations "psys" "agrémentent", à défaut pour certains auteurs d'avoir rencontré des «adeptes». Dericquebourg montre plusieurs modes de «mise hors norme » des GRM utilisés dans ces livres : l'absurdité ou la fausseté des croyances, la " victimisation » des «fidèles ", la dépréciation de leur santé mentale, également celle du « gourou » considéré « pathogène » de surcroît, et la « criminalisation » des groupes qui rejoint leur «pathologisation » comme si la nosographie humaine pouvait prendre corps dans un mouvement social. Cette rhétorique entend décrire un danger à tous les niveaux, de l'individuel au sociétal, dont l'auteur déduit d'une part que les «psys » ne relèvent pas des sciences, et d'autre part que leurs discours sont "une réécriture contre l'hérésie et contre les sorcières qui véhiculent une normalité religieuse ». Aussi grave, sur les plans méthodologique et éthique, est «l'absence de décentrement par rapport à l'objet » (p. 117) ou le manque de distance, qui vient consolider une certaine réalité sociale, adossée à une répression du non-conformisme.

8 En guise de conclusion, le chapitre de l'avocat et sociologue Dominique Kounkou porte sur la notion de « liberté religieuse » (chap. 7). «Liberté politique » car subordonnée au pouvoir, elle n'est pas sans lien avec la laïcité qui, différemment appliquée dans l'espace public, le champ judiciaire et à l'école, montre ses limites en France où « toutes les religions ne se valent pas aux yeux de l'État» (p.144). Pour Dericquebourg " [1]'échec du modèle français des libertés religieuses tient à leur qualification par les libertés publiques» (p.148). Ces dernières, comme les Droits de l'Homme ou la Déclaration de Virginie, accordent peu de place aux premières, et s'arrogent la fonction de prescrire les normes de vie humaine. Face à ce "choix politique ", l'auteur affirme au final que " [la] métamorphose de l'idée de liberté religieuse située à ce rang [des libertés politiques décrites par les libertés publiques] est de très haute importance pour l'ensemble de l'action politique, surtout lorsque les acteurs d'un État comme la France pratique une politique moniste qui s'épargne de toute idée de l'intervention de Dieu dans la vie de citoyens » (p. 151).

9 Au final, cet ouvrage consacré à l'étude des rapports antagoniques aux " hérétiques » suggère aussi de nouvelles pistes d'analyse, comme les coalitions antisectes ellesmêmes : la Miviludes et l'Unadfi qui s'associent à la Franc-maçonnerie alors que celle-ci est accusée par d'autres d'être une "secte ", et aux «psys » dans une perspective qui privilégierait l'aspect marchand. Mais il ne faut pas oublier non plus le rapprochement de plus en plus flagrant des GRM avec le droit international, et leur référence désormais fréquente aux Droits de l'Homme en particulier. Espérons que ces quelques pistes raviveront l'attention des sciences sociales des religions à l'égard des dites «sectes ", à moins que la recherche ne s'ouvre à d'autres champs où ces dernières ou les « hérétiques » trouveront de l'intérêt. 